(C) 2021 IEEE. Personal use of this material is permitted. Permission from IEEE must be obtained for all other uses, in any current or future media, including

reprinting/republishing this material for advertising or promotional purposes, creating new collective works, for resale or redistribution to servers or lists, or reuse of any copyrighted component of this work in other works. 


\title{
Semi-Grant-Free Non-Orthogonal Multiple Access for Tactile Internet of Things
}

\author{
Dimitrios Pliatsios*, Alexandros-Apostolos A. Boulogeorgos ${ }^{\dagger}$, Thomas Lagkas ${ }^{\S}$, \\ Vasileios Argyriou ${ }^{\top}$, Ioannis D. Moscholios ${ }^{\ddagger}$, and Panagiotis Sarigiannidis* \\ * Department of Electrical and Computer Engineering, University of Western Macedonia, Kozani 50100, Greece \\ $\dagger$ Department of Digital Systems, University of Piraeus, Piraeus 18534, Greece \\ $\S$ Department of Computer Science, International Hellenic University, Kavala Campus, Greece \\ ฯ Kingston University London, SEC, Kingston, London KT12EE, United Kingdom \\ $\ddagger$ Department Informatics \& Telecommunications, University of Peloponnese, Tripolis 22100, Greece \\ dpliatsios@uowm.gr, al.boulogeorgos@ieee.org, tlagkas@cs.ihu.gr, \\ Vasileios.Argyriou@kingston.ac.uk, idm@uop.gr, psarigiannidis@uowm.gr
}

\begin{abstract}
Ultra-low latency connections for a massive number of devices are one of the main requirements of the nextgeneration tactile Internet-of-Things (TIoT). Grant-free nonorthogonal multiple access (GF-NOMA) is a novel paradigm that leverages the advantages of grant-free access and non-orthogonal transmissions, to deliver ultra-low latency connectivity. In this work, we present a joint channel assignment and power allocation solution for semi-GF-NOMA systems, which provides access to both grant-based (GB) and grant-free (GF) devices, maximizes the network throughput, and is capable of ensuring each device's throughput requirements. In this direction, we provide the mathematical formulation of the aforementioned problem. After explaining that it is not convex, we propose a solution strategy based on the Lagrange multipliers and subgradient method. To evaluate the performance of our solution, we carry out system-level Monte Carlo simulations. The simulation results indicate that the proposed solution can optimize the total system throughput and achieve a high association rate, while taking into account the minimum throughput requirements of both GB and GF devices.
\end{abstract}

Index Terms-Grant-Free, Internet of Things, Non-Orthogonal Multiple Access

\section{INTRODUCTION}

The proliferation of tactile Internet-of-Things (TIoT) devices along with the emergence of new application domains, such as industry 4.0 [1], healthcare [2], transportation [3], and agriculture [4], that demand high-reliability, low-latency and massive connectivity [5]. Consequently, these new application domains are expected to stretch access networks to their limits [6], [7]. Aspired by this, both the research and development communities turned their attention to the design and analysis of new non-orthogonal multiple access (NOMA) schemes (see e.g., [8]-[12] and references therein).

Although GF schemes are able to achieve the low-latency requirements, they lack in reliability in TIoT environments where a massive number of devices may need to be supported. To address this, GF-NOMA approaches have been presented and examined as possible solutions [13]-[17]. Particularly, in [13], the authors derived simplified expressions that approximate the outage probability and system throughout for both successive joint decoding (SJD) and successive interference cancellation (SIC), while in [14], Du et al. developed an algorithm that exploits the block sparsity to effectively carry out the multi-user detection (MUD) process. Jiang et al. [15] presented a distributed transmission scheme that aims to mitigate collisions in massive machine-type communications (mMTC) scenarios, while, in [16], they developed a joint user and signal detection algorithm by leveraging the message passing principles of GF-NOMA systems. Finally, in [17], the authors utilized a deep neural network to develop an active user detection (AUD) method for mMTC.

The aforementioned works presented some interesting results; however, they present resource allocation policies for scenarios in which only GF devices are involved. However, in practice, GF and GB devices coexist and are expected to be served by the same base station (BS). In light of the aforementioned remark, we present a novel semi-GF-NOMA solution that ensures connectivity for both critical and noncritical IoT devices. Critical IoT devices require highly reliable links to the BS, and thus, they are allocated dedicated channels. On the other hand, non-critical devices have lower reliability requirements. These devices send their data in a GF manner, if and only if the interference that they cause does not degrade the performance of the critical IoT devices. In more detail, the technical contribution of this paper is as follows:

- We present a general system model for TIoT networks, in which both GF and GB devices of different throughput requirements coexist.

- We formulate a total throughput maximization problem as a joint channel assignment and power allocation problem.

- After explaining that the aforementioned problem is not convex, we propose a solution strategy based on the Lagrange multipliers and subgradient method.

- We analyze the complexity of the proposed solution.

- To evaluate the performance of the proposed solution, we carry out extensive system-level Monte Carlo simulations.

The rest of the paper is structured as follows: Section II introduces the system model. The formulation and solution of the optimization problem is presented in Section III, while 


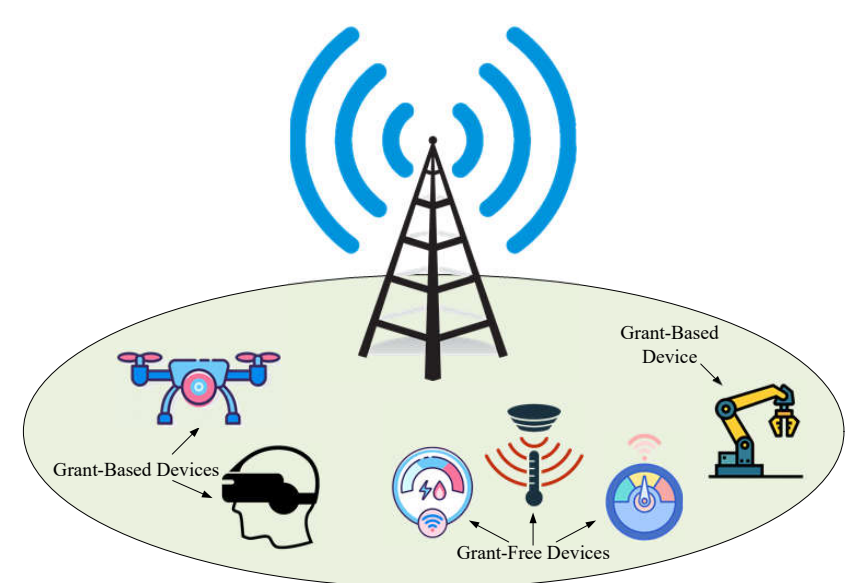

Fig. 1. Uplink Semi-GF-NOMA Scenario

Section IV presents simulation results and insightful discussions. Finally, Section V concludes this work.

\section{SySTEM MODEL}

As depicted in Fig. 1, we consider an uplink scenario, where a BS provides radio access to a number of $N$ randomly deployed devices. There are two categories of devices, namely the GB devices, which are have already been assigned a channel, and the GF devices that intend to share the channels with the GB devices. To support massive connectivity and increased spectrum efficiency, there are $M$ channels available for both GB and GF transmission. Furthermore, it is assumed that up to one GF device can be assigned to the same channel.

The SIC process is utilized for MUD. Each device is aware of its own channel state information (CSI), as well as its maximum available transmission power. Likewise, GF devices "eavesdrop" the communication between the GB devices and the BS, in order to gain knowledge of the GB devices CSI. Moreover, the GF devices also autonomously communicate with each other, based on a device-to-device (D2D) communication scheme, in order to exchange their CSI and throughput requirements. To achieve this alternative frequency bands are utilized for D2D communications, such as millimeter-wave or microwave frequencies [18], [19]. As a result, the signaling overhead between the GF devices and the BS is minimized.

Let the gain of device $n$ over channel $m$ be denoted by $\left|h_{n, m}\right|$. The term $p_{n, m}$ stands for the transmission power of device $n$ to channel $m$. Without loss of generality, we assume that the number of channels $M$ equals the number of GB devices and the GB devices are ordered based on their respective channel gains as $\left|h_{1, m}\right| \geq\left|h_{2, m}\right| \geq \ldots \geq\left|h_{M, m}\right|, \forall m$. The channel coefficients are assumed to follow ordered Rayleigh distribution with variance $d_{n}^{-2}$, where $d_{n}$ is the BS to the $n$ th device distance. Both GF and GB devices have different throughput requirements, denoted by $R_{n}^{\min }, 1 \leq n \leq N$. Finally, the binary variable $a_{n, m}$ denotes whether device $n$ is assigned to channel $m$.

Based on the decoding order of the SIC process, i.e. lower-ordered devices will not introduce interference to the considered device, the signal-to-interference-plus-noise ratio (SINR) of device $n$ in channel $m$ is

$$
\gamma_{n, m}=\frac{\left|h_{n, m}\right|^{2} p_{n, m}}{\left|h_{n, m}\right|^{2} \sum_{l=n+1}^{N} p_{l, m}+1}
$$

Note that without loss of generality, we assume that the noise power is equal to 1 . Consequently, the achievable throughput of device $n$ in channel $m$ can be calculated as:

$$
R_{n, m}=\log _{2}\left(1+\gamma_{n, m}\right)
$$

Finally, the GB devices have already been assigned to their respective channels, where they feature the best performance, hence, the binary assignment variable will be:

$$
a_{n, m}=\left\{\begin{array}{ll}
1, & \text { if } n=m \\
0, & \text { otherwise }
\end{array} \forall n, m\right.
$$

\section{Joint Channel AND Power Allocation}

The solution to the joint channel assignment and power allocation problem is presented in this section. In particular, this section provides the problem formulation and the corresponding solution policy.

\section{A. Problem Formulation}

We propose a joint optimization solution that aims to maximize the total system throughput. The proposed solution determines the optimal GF device assignment to a channel, as well as its optimal transmission power in the assigned channel so as to minimize the interference to the GB device. In this direction, the optimization problem can be formulated as

$$
\max _{a_{n, m}, p_{n, m}} \sum_{n=1}^{N} \sum_{m=1}^{M} a_{n, m} R_{n, m}
$$

subject to:

$$
\begin{aligned}
\mathcal{C} 1 & : a_{n, m} R_{n, m} \geq R_{n}^{\text {min }}, \forall n, m \\
\mathcal{C} 2 & : \sum_{m=1}^{M} p_{n, m} \leq P_{n}^{\text {max }}, 1 \leq n \leq N \\
\mathcal{C} 3: & \sum_{n=1}^{N} a_{n, m} \leq 2,1 \leq m \leq M \\
\mathcal{C} 4: & \sum_{n=1}^{M} \sum_{m=1}^{M} a_{n, m} \leq M \\
\mathcal{C} 5 & : \sum_{m=1}^{M} a_{n, m} \leq 1,1 \leq n \leq N \\
\mathcal{C} 6 & : a_{n, m}=\{0,1\}, \forall n, m \\
\mathcal{C} 7: & p_{n, m} \geq 0, \forall n, m
\end{aligned}
$$

In (4), $\mathcal{C} 1$ indicates that each device is required to achieve its minimum required throughput, while $\mathcal{C} 2$ limits the transmission power to the maximum transmission power of each device. If more than two devices are assigned to the same channel, the performance of the SIC process is dramatically reduced [20]-[22]. To this end, $\mathcal{C} 3$ limits the number of 
assigned devices per channel to two devices. $\mathcal{C} 4$ and $\mathcal{C} 5$ are imposed to guarantee that the already assigned GB devices will not be assigned to different channels. Finally, $\mathcal{C} 6$ enforces binary values to $a_{n, m}$.

The optimization problem in (4) is a non-convex mixed integer problem, due to the binary variable $a_{n, m}$. To solve it, we first relax $\mathcal{C} 6$ as

$$
\mathcal{C} 6: 0 \leq a_{n, m} \leq 1, \forall n, m
$$

and we employ the Lagrange multipliers method [23]. The Lagrangian of (4) can be obtained as (5), given at the top of the following page.

In (5), $\lambda_{n, m}, \xi_{n}, \theta_{m}, \kappa, \phi_{n}$, and $\chi_{n, m}$ are the non-negative Lagrange multipliers corresponding to the optimization constraints. By defining $\mathcal{X}=\left(a_{n, m}, p_{n, m}, \lambda_{n, m}, \xi_{n}, \theta_{m}, \kappa, \phi_{n}, \chi_{n, m}\right)$ in (5), we extract

$$
\begin{gathered}
\mathcal{L}(\mathcal{X})=\sum_{n=1}^{N} \sum_{m=1}^{M} a_{n, m}\left(R_{n, m}+\lambda_{n, m} R_{n, m}\right. \\
\left.-\theta_{m}-\phi_{n}-\chi_{n, m}\right)-\xi_{n} p_{n, m}+\chi_{n, m} \\
+\sum_{n=1}^{N}-\lambda_{n, m} R_{n}^{m i n}+\xi_{n} P_{n}^{\max }+\phi_{n} \\
+2 \sum_{m=1}^{M} \theta_{m}+\kappa M-\kappa \sum_{n=1}^{M} \sum_{m=1}^{M} a_{n, m}
\end{gathered}
$$

which can equivalently written as

$$
\begin{aligned}
& \mathcal{L}(X)=\sum_{n=1}^{N} \sum_{m=1}^{M} L_{n, m}(X) \\
& +\sum_{n=1}^{N}\left(-\lambda_{n, m} R_{n}^{\text {min }}+\xi_{n} P_{n}^{\max }+\phi_{n}\right) \\
& \quad+\sum_{m=1}^{M}\left(2 \theta_{m}\right)+\kappa M
\end{aligned}
$$

where

$$
\begin{aligned}
& L_{n, m}(X)=a_{n, m}\left(R_{n, m}+\lambda_{n, m} R_{n, m}-\theta_{m}-\phi_{n}-\right. \\
& \left.\chi_{n, m}-\kappa\right)-\xi_{n} p_{n, m}+\chi_{n, m}+\frac{k}{N} \sum_{y=M+1}^{N} a_{y, m}
\end{aligned}
$$

Consequently, the dual problem in (4) can be equivalently expressed as

$$
\begin{array}{r}
\min _{\lambda_{n, m}, \xi_{n}, \theta_{m}, \kappa, \phi_{n}, \chi_{n, m}} D\left(\lambda_{n, m}, \xi_{n}, \theta_{m}, \kappa, \phi_{n}, \chi_{n, m}\right) \\
\text { subject to: } \lambda_{n, m}, \xi_{n}, \theta_{m}, \kappa, \phi_{n}, \chi_{n, m} \geq 0
\end{array}
$$

where the dual function is defined as

$$
D\left(\lambda_{n, m}, \xi_{n}, \theta_{m}, \kappa, \phi_{n}, \chi_{n, m}\right)=\max _{\left\{a_{n, m}\right\},\left\{p_{n, m}\right\}} \mathcal{L}(X)
$$

The aforementioned dual problem in (4) can be decomposed into two layers, namely the inner and outer layers. Specifically, in the inner layer, the optimal channel assignment and power allocation (i.e. $a_{n, m}$ and $p_{n, m}$ ) are determined, while in the outer layer, the optimal Lagrange multipliers (i.e. $\lambda_{n, m}, \xi_{n}$, $\theta_{m}, \kappa, \phi_{n}$ and $\left.\chi_{n, m}\right)$ are calculated.

The following theorem returns the optimal power allocation policy for the GF devices.

Theorem 1. The optimal power allocation can be obtained as

$p_{n, m}^{*}=\left[\frac{a_{n, m}\left(1+\lambda_{n, m}\right)}{\xi_{n} \ln 2}-\frac{1+\left|h_{n, m}\right|^{2}\left(\sum_{l=n+1}^{N} p_{l, m}\right)}{\left|h_{n, m}\right|^{2}}\right]^{+}$

where $[x]^{+}=\max (0, x)$.

Proof: For brevity, the proof is provided in the Appendix.

To obtain the allocation variable, we calculate the partial derivative of the Lagrangian as

$$
\frac{\partial \mathcal{L}_{n, m}(X)}{\partial a_{n, m}}=\Delta_{n, m}-\theta_{m}-\phi_{n}-\chi_{n, m}-K
$$

where

$$
\Delta_{n, m}=\left(1+\lambda_{n, m}\right) R_{n, m}-\xi_{n} p_{n, m}
$$

and

$$
K=\left\{\begin{array}{l}
\kappa, \text { if } n \leq M \\
\frac{4 \kappa}{5}, \text { if } n>M
\end{array}\right.
$$

Therefore, the optimal IoT device-channel association can be obtained as

$$
a_{n, m^{*}}=1 \mid m^{*}=\max _{m} \frac{\partial \mathcal{L}_{n, m}(X)}{\partial a_{n, m}}, \forall n
$$

Note that, as $a_{n, m}$ is set to 0 or 1 according to (15), the relaxation of $\mathcal{C} 6$ does not have any impact in the optimality of the solution.

Based on the subgradient method (Chapter 2 of [24]) the Lagrange multipliers can be updated as

$$
\begin{aligned}
\lambda_{n, m}^{t+1} & =\left[\lambda_{n, m}^{t}-s_{1}\left(R_{n, m} a_{n, m}-R_{n}^{\text {min }}\right)\right]^{+} \\
\xi_{n}^{t+1} & =\left[\xi_{n}^{t}-s_{2}\left(P_{n}^{\max }-\sum_{m=1}^{M} p_{n, m}\right)\right]^{+} \\
\theta_{m}^{t+1} & =\left[\theta_{m}^{t}-s_{3}\left(2-\sum_{n=1}^{N} a_{n, m}\right)\right]^{+} \\
\kappa^{t+1} & =\left[\kappa^{t+1}-s_{4}\left(M-\sum_{n=1}^{N} \sum_{m=1}^{M} a_{n, m}\right)\right]^{+} \\
\phi_{n}^{t+1} & =\left[\phi_{n}^{t}-s_{5}\left(1-\sum_{m=1}^{M} a_{n, m}\right)\right]^{+} \\
\chi_{n, m}^{t+1} & =\left[\chi_{n, m}^{t}-s_{6}\left(1-a_{n, m}\right)\right]^{+}
\end{aligned}
$$

where $t$ denotes the iteration index, while $s_{1}, s_{2}, s_{3}, s_{4}, s_{5}$, and $s_{6}$ are the positive step sizes. 


$$
\begin{aligned}
& \mathcal{L}\left(a_{n, m}, p_{n, m}, \lambda_{n, m}, \xi_{n}, \theta_{m}, \kappa, \phi_{n}, \chi_{n, m}\right)=\sum_{n=1}^{N} \sum_{m=1}^{M} R_{n, m} a_{n, m}+\sum_{n=1}^{N} \sum_{m=1}^{M} \lambda_{n, m}\left(R_{n, m} a_{n, m}-R_{n}^{\text {min }}\right) \\
& +\sum_{n=1}^{N} \xi_{n}\left(P_{n}^{\max }-\sum_{m=1}^{M} p_{n, m}\right)+\sum_{m=1}^{M} \theta_{m}\left(2-\sum_{n=1}^{N} a_{n, m}\right)+\kappa\left(M-\sum_{n=1}^{M} \sum_{m=1}^{M} a_{n, m}\right) \\
& +\sum_{n=1}^{N} \phi_{n}\left(1-\sum_{m=1}^{M} a_{n, m}\right)+\sum_{n=1}^{N} \sum_{m=1}^{M} \chi_{n, m}\left(1-a_{n, m}\right)
\end{aligned}
$$

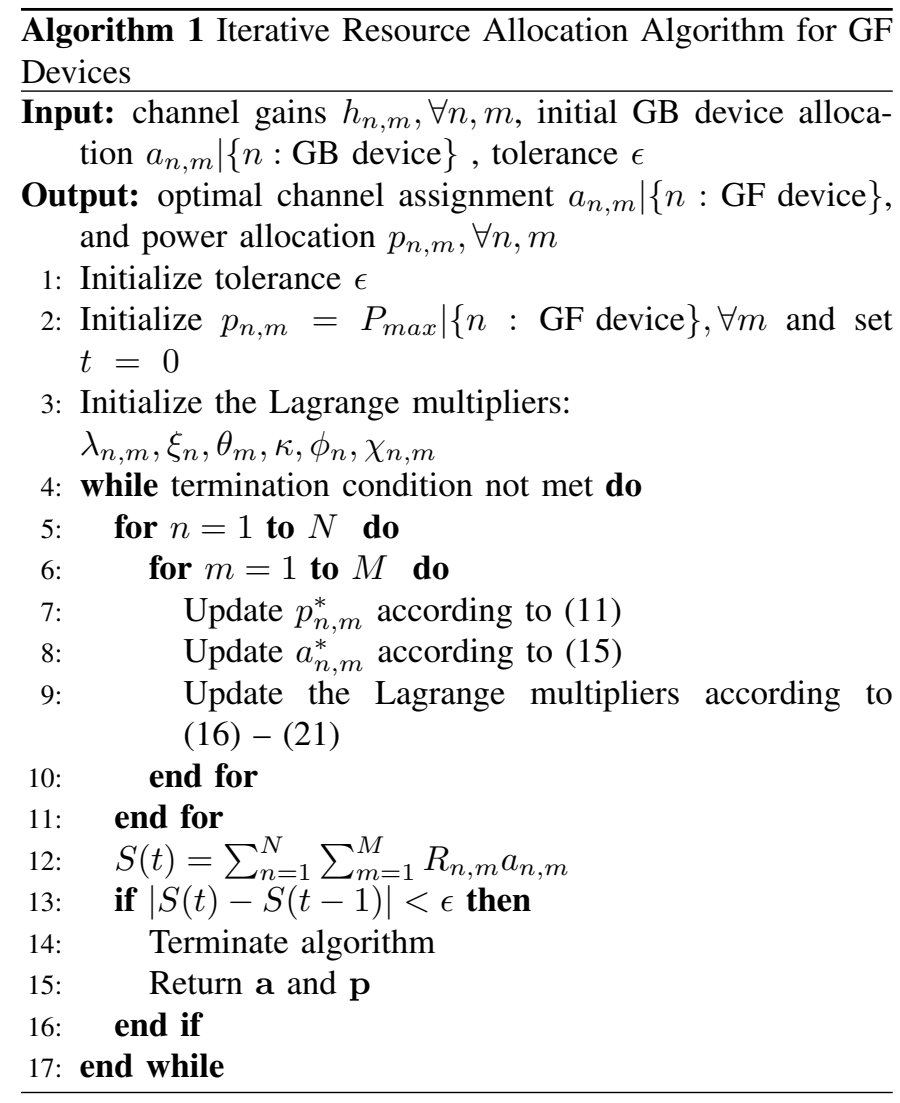

\section{B. Solution Policy}

In this section, we present Algorithm 1 that returns the optimal joint channel assignment and power allocation of GF devices. Algorithm 1 uses as inputs the channel gains, the GB device assignment, and the tolerance, as the termination criterion. The total network throughput increases after each iteration until convergence and the algorithm outputs the optimal GF channel assignment and power allocation. At each iteration, given the total throughput from the last iteration, the optimal channel assignment and power allocation for each GF device will be updated until convergence.

In Algorithm 1, we suppose the iteration number converges at $T$. In each iteration of the inner layer, the calculation of (11) entails $N M$ operations, and the worst-case complexity of searching (14) requires $N M$ operations in each iteration of the inner layer. The updates of the Lagrange multipliers (15)-(16)

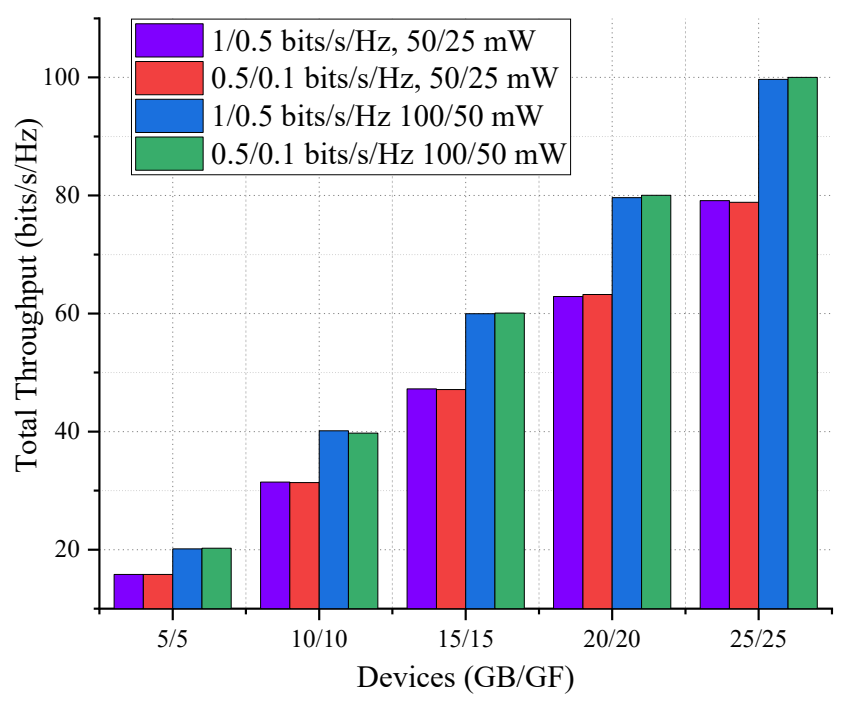

Fig. 2. Total throughput as a function of the number of devices for different throughput requirements and maximum transmission power.

require $O(N M)$ operations. Therefore, the total complexity of Algorithm 1 is $O(T N M)$. Additionally, with the appropriate selection of iteration step sizes $\left(s_{1}, \ldots, s_{6}\right), T$ can be further reduced.

\section{PERformance Evaluation}

To evaluate the performance of our proposed solution, we utilize extensive Monte Carlo simulations. Specifically, we evaluate the total system throughput and association rate as functions of the number of devices for different throughput requirements and maximum available transmission power. In more detail the following insightful scenario is investigated. We assume that the GB and GF devices are randomly distributed in the coverage region, while their distances from the BS are in the range of $[1,10] \mathrm{m}$. Additionally, the number of channels is assumed to be equal to the number of GB devices. The tolerance is set to $\epsilon=10^{-3}$, while the step sizes $\left(s_{1}-s_{6}\right)$ are set to 0.1 .

Figure 2 shows the total throughput as a function of the number of devices, as the number of both GB and GF devices is $5,10,15,20$, and 25 . The minimum throughput requirements are 1 and $0.5 \mathrm{bps} / \mathrm{s} / \mathrm{Hz}$ for GB devices, while the respective throughput requirements for GF devices are 0.5 and $0.1 \mathrm{bps} / \mathrm{s} / \mathrm{Hz}$. Similarly, the maximum transmission 


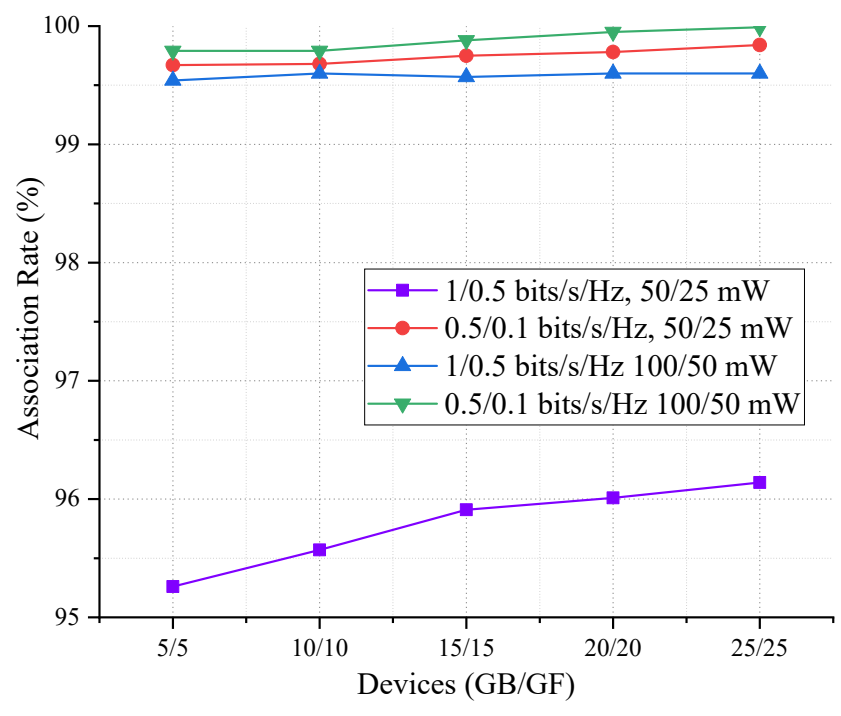

Fig. 3. Association rate as a function of the number of devices for different throughput requirements and maximum transmission power

power is 50 and $100 \mathrm{~mW}$ for GB devices, while the respective maximum transmission power of GF devices are 25 and 50 $\mathrm{mW}$. It is apparent that the total throughput is increased as the number of GB and GF devices increases. Similarly, when the maximum available power is increased from 50/25 to $100 / 50$ $\mathrm{mW}$ the total throughput also increases. This is expected as both GB and GF devices transmit with more power, thus, they are able to achieve higher throughput. Finally, according to the results, the minimum throughput requirement does not have an impact on the total throughput. This is expected, the proposed algorithm optimizes the total system throughput. As a result, the achieved device throughput is higher than the minimum required one.

Figure 3 illustrates the association rate of GF devices as a function of the total number of devices. Of note, a GF device is successfully associated when its minimum throughput requirement is satisfied. The minimum throughput requirements are 1 and $0.5 \mathrm{bps} / \mathrm{s} / \mathrm{Hz}$ for GB devices, while the respective throughput requirements for GF devices are 0.5 and 0.1 $\mathrm{bps} / \mathrm{s} / \mathrm{Hz}$. Similarly, the maximum available power is 50 and $100 \mathrm{~mW}$ for GB devices and 25 and $50 \mathrm{~mW}$ for GF devices, respectively. As the maximum available power is increased, the association rate can achieve values higher than $99.5 \%$. This is expected, as GF devices can achieve a higher throughput, when more power is available. A similar association rate is achieved when the minimum throughput requirements are lower, as the maximum available power is sufficient to achieve the required throughput. Finally, an association rate of about $96 \%$ is achieved when the throughput requirements are higher, but the maximum available power is low.

Figure 4 illustrates the convergence of Algorithm 1 for different numbers of GB and GF devices, as well as different values of maximum transmission power. When there are fewer GB and GF devices, Algorithm 1 converges after 30 iterations. On the other hand, when there is a high number of devices,

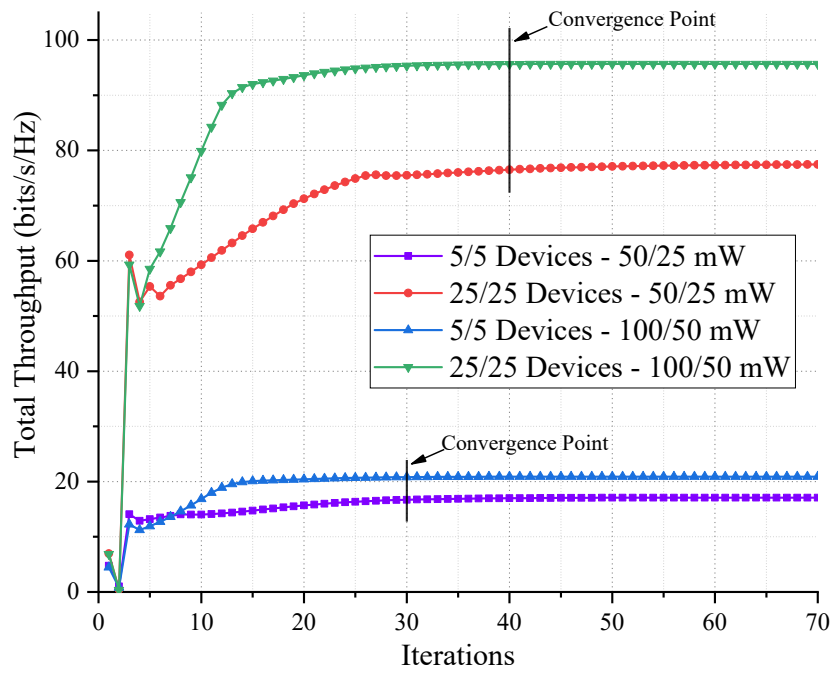

Fig. 4. Convergence of Algorithm 1

Algorithm 1 converges after 40 iterations. This is expected, as, in case there are more devices, there exist more channel-device combinations to be considered. Furthermore, the maximum transmission power does not noticeably affect the convergence speed. Finally, note that the spikes at the first and second iterations are expected, because the initial transmission power of GB devices is set to P $\max$.

\section{CONCLUSion}

In this work, we proposed a joint channel assignment and power allocation solution for GF-NOMA network. The proposed solution aims to optimally assign channels to the GF devices, as well as optimize the allocated power of both GF and GB devices. We utilized Lagrange dual decomposition to relax the initial non-convex optimization problem. We derived closed-form expressions for the optimal power allocation and designed a low-complexity algorithm that iteratively finds the optimal channel allocation for GF devices.

To validate the efficiency of the proposed solution, we carried out system-level Monte Carlo simulations and evaluated the total system throughput and association rate. The simulation results showed that the proposed solution can maximize the total system throughput and maintain a high association rate while taking into account the throughput requirements of each device. Finally, the complexity analysis shows that our proposed solution features a low complexity. This can be also validated by the respective results concerned with convergence speed of Algorithm 1.

\section{APPENDIX \\ PROOF OF THEOREM 1}

For simplicity, let us assume that $N=3$. In this case, for any channel $m$, we assume that the devices are sorted according to the channel gains $\left|h_{1, m}\right| \geq\left|h_{2, m}\right| \geq\left|h_{3, m}\right|$. To obtain $p_{1, m}^{*}$, we calculate the derivative of (8) as

$$
\frac{\partial L_{1, m}}{\partial p_{1, m}}=0
$$


or

$$
-\xi_{1}+\frac{a_{1, m}\left(1+\lambda_{1, m}\right)\left|h_{1, m}\right|^{2}}{\ln 2\left(1+\left|h_{1, m}\right|^{2}\left(p_{1, m}+p_{2, m}+p_{3, m}\right)\right)}=0
$$

which can be rewritten as

$$
p_{1, m}^{*}=\frac{\left(1+\lambda_{1, m}\right)}{\xi_{1} \ln 2}-\frac{1}{\left|h_{1, m}\right|^{2}}-\left(p_{2, m}+p_{3, m}\right)
$$

Similarly, to obtain $p_{2, m}^{*}$

$$
\frac{\partial L_{2, m}}{\partial p_{2, m}}=0
$$

or

$$
-\xi_{2}+\frac{a_{2, m}\left(1+\lambda_{2, m}\right)\left|h_{2, m}\right|^{2}}{\ln 2\left(1+\left|h_{2, m}\right|^{2}\left(p_{2, m}+p_{3, m}\right)\right)}=0
$$

which can be rewritten as

$$
p_{2, m}^{*}=\frac{a_{2, m}\left(1+\lambda_{2, m}\right)}{\xi_{2} \ln 2}-\frac{1}{\left|h_{2, m}\right|^{2}}-\left(p_{3, m}\right)
$$

Finally, to obtain $p_{3, m}^{*}$

$$
\frac{\partial L_{3, m}}{\partial p_{3, m}}=0
$$

or

$$
-\xi_{3}+\frac{a_{3, m}\left(1+\lambda_{3, m}\right)\left|h_{3, m}\right|^{2}}{\ln 2\left(1+\left|h_{3, m}\right|^{2} p_{3, m}\right)}=0
$$

which can be rewritten as

$$
p_{3, m}^{*}=\frac{a_{3, m}\left(1+\lambda_{3, m}\right)}{\xi_{3} \ln 2}-\frac{1}{\left|h_{3, m}\right|^{2}}
$$

Through deduction and taking into account (24), (27), and (30), the optimal power allocated to device $n$ on channel $m$ can be derived by (11).

\section{ACKNOWLEDGMENT}

This research is co-financed by Greece and the European Union (European Social Fund- ESF) through the Operational Programme "Human Resources Development, Education and Lifelong Learning" in the context of the project "Strengthening Human Resources Research Potential via Doctorate Research" (MIS-5000432), implemented by the State Scholarships Foundation (IKY).

This work has received funding from the European Union's Horizon 2020 research and innovation programme under grant agreement No. 957406 (TERMINET).

\section{REFERENCES}

[1] A.-A. A. Boulogeorgos, P. D. Diamantoulakis, and G. K. Karagiannidis, "Low power wide area networks (lpwans) for internet of things (iot) applications: Research challenges and future trends," arXiv preprint arXiv:1611.07449, 2016.

[2] Y. Miao, Y. Jiang, L. Peng, M. S. Hossain, and G. Muhammad, "Telesurgery robot based on 5g tactile internet," Mobile Networks and Applications, vol. 23, no. 6, pp. 1645-1654, Sep 2018.

[3] V. Chauhan, M. Patel, S. Tanwar, S. Tyagi, and N. Kumar, "IoT enabled real-time urban transport management system," Computers \& Electrical Engineering, vol. 86, p. 106746, Sep 2020.

[4] A. Lytos, T. Lagkas, P. Sarigiannidis, M. Zervakis, and G. Livanos, "Towards smart farming: Systems, frameworks and exploitation of multiple sources," Computer Networks, vol. 172, p. 107147, May 2020.
[5] A. N., K. H. J. Rai, H. M.K., R. Ramesh, R. Hegde, and S. Kumar, "Tactile internet: Next generation IoT," in Third International Conference on Inventive Systems and Control (ICISC), IEEE. IEEE, Jan 2019, pp. 22-26.

[6] D. Pliatsios, P. Sarigiannidis, S. Goudos, and G. K. Karagiannidis, "Realizing 5g vision through cloud RAN: technologies, challenges, and trends," EURASIP Journal on Wireless Communications and Networking, vol. 2018, no. 1, p. 136, May 2018.

[7] P. Sarigiannidis, T. Lagkas, S. Bibi, A. Ampatzoglou, and P. Bellavista, "Hybrid 5g optical-wireless SDN-based networks, challenges and open issues," IET Networks, vol. 6, no. 6, pp. 141-148, Nov 2017.

[8] N. H. Mahmood, R. Abreu, R. Bohnke, M. Schubert, G. Berardinelli, and T. H. Jacobsen, "Uplink grant-free access solutions for URLLC services in $5 \mathrm{~g}$ new radio," in 16th International Symposium on Wireless Communication Systems (ISWCS), IEEE. IEEE, Aug 2019, pp. 607612.

[9] D. Pliatsios and P. Sarigiannidis, "Resource allocation combining heuristic matching and particle swarm optimization approaches: The case of downlink non-orthogonal multiple access," Information, vol. 10, no. 11, p. 336, Oct 2019.

[10] M. B. Shahab, R. Abbas, M. Shirvanimoghaddam, and S. J. Johnson, "Grant-free non-orthogonal multiple access for IoT: A survey," IEEE Communications Surveys \& Tutorials, vol. 22, no. 3, pp. 1805-1838, 2020.

[11] S. A. Tegos, P. D. Diamantoulakis, A. S. Lioumpas, P. G. Sarigiannidis, and G. K. Karagiannidis, "Slotted ALOHA with NOMA for the next generation IoT," IEEE Transactions on Communications, vol. 68, no. 10, pp. 6289-6301, Oct 2020.

[12] J. Li, X. Lei, P. D. Diamantoulakis, F. Zhou, P. Sarigiannidis, and G. K. Karagiannidis, "Resource allocation in buffer-aided cooperative non-orthogonal multiple access systems," IEEE Transactions on Communications, pp. 1-1, 2020.

[13] R. Abbas, M. Shirvanimoghaddam, Y. Li, and B. Vucetic, "A novel analytical framework for massive grant-free NOMA," IEEE Transactions on Communications, vol. 67, no. 3, pp. 2436-2449, Mar 2019.

[14] Y. Du, B. Dong, W. Zhu, P. Gao, Z. Chen, X. Wang, and J. Fang, "Joint channel estimation and multiuser detection for uplink grant-free NOMA," IEEE Wireless Communications Letters, vol. 7, no. 4, pp. 682685, Aug 2018.

[15] H. Jiang, Q. Cui, Y. Gu, X. Qin, X. Zhang, and X. Tao, "Distributed layered grant-free non-orthogonal multiple access for massive MTC," in IEEE 29th Annual International Symposium on Personal, Indoor and Mobile Radio Communications (PIMRC), IEEE. IEEE, Sep 2018, pp. $1-7$.

[16] S. Jiang, X. Yuan, X. Wang, and C. Xu, "Joint user identification, channel estimation, and signal detection for grant-free NOMA," IEEE Transactions on Wireless Communications, Dec 2019.

[17] W. Kim, Y. Ahn, and B. Shim, "Deep neural network-based active user detection for grant-free NOMA systems," IEEE Transactions on Communications, vol. 68, no. 4, pp. 2143-2155, Apr 2020.

[18] N. Giatsoglou, K. Ntontin, E. Kartsakli, A. Antonopoulos, and C. Verikoukis, "D2d-aware device caching in mmWave-cellular networks," IEEE Journal on Selected Areas in Communications, vol. 35, no. 9, pp. 20252037, sep 2017.

[19] S. Wu, R. Atat, N. Mastronarde, and L. Liu, "Improving the coverage and spectral efficiency of millimeter-wave cellular networks using device-to-device relays," IEEE Transactions on Communications, vol. 66, no. 5, pp. 2251-2265, may 2018.

[20] M. Qu, J. Liu, J.-B. Seo, and H. Jin, "Distributed fair channel access in NOMA random access systems," in IEEE Global Communications Conference (GLOBECOM), IEEE. IEEE, Dec 2019, pp. 1-6.

[21] A.-A. A. Boulogeorgos, N. D. Chatzidiamantis, and G. K. Karagiannidis, "Non-orthogonal multiple access in the presence of phase noise," IEEE Communications Letters, vol. 24, no. 5, pp. 1133-1137, May 2020.

[22] H. Zhang, H. Zhang, K. Long, and G. Karagiannidis, "Deep learning based radio resource management in NOMA networks: User association, subchannel and power allocation," IEEE Transactions on Network Science and Engineering, pp. 1-1, 2020.

[23] S. Boyd, S. P. Boyd, and L. Vandenberghe, Convex optimization. Cambridge university press, 2004.

[24] N. Z. Shor, Minimization methods for non-differentiable functions. Springer Science \& Business Media, 2012, vol. 3. 